\title{
Operator Decision Making in the Minerals Industry
}

\author{
Tim Horberry ${ }^{*}$, Tristan Cooke, Xilin Li and Garry Marling
}

Minerals Industry Safety \& Health Centre, University of Queensland, QLD 4072, Australia

\begin{abstract}
The minerals industry is a complex work domain where people, procedures and equipments need to interact safely and efficiently. Given the importance of the human element in this industry, it is surprising that, to date, comparatively few studies have been published that specifically examine operator decision making. This paper presents two ongoing case studies from different sectors of mining and minerals processing that are drawing heavily on 'Naturalistic Decision Making' (NDM) approaches and methods. The case studies involve analyzing incidents using the Critical Decision Method and how Naturalistic Decision Making methods and design processes can help improve interfaces in process control. Following this, the paper will discuss this work and comment on the worth of the overall NDM approach to the minerals industry. Some of the lessons learnt will be highlighted and potential future research recommended.
\end{abstract}

Keywords: Human factors, naturalistic decision making, minerals industry, operator-centred design, incident investigation.

\section{INTRODUCTION: NATURALISTIC DECISION MAKING IN THE MINERALS INDUSTRY}

Naturalistic Decision Making (NDM) is the study of how operators make decisions in real world settings [1], and how the study of such decisions can then be used, for example, to help investigate incidents, develop better training for novices or design better systems. It found that, in real situations, experienced operators generated a very limited number, even single, possible course of action, and then they compared this to the constraints of the situation by means of matching cues and patterns within a situation to play out action scripts [2]. NDM finds that people often use mental shortcuts, also known as 'rules of thumb' or heuristics, to make decisions, with experts having developed more effective heuristics with experience and repetition of successful outcomes. More often these shortcuts are taken by experts when time is critical.

Over the past 20 years, most NDM research to date has been undertaken in the military, medical or firefighting domains. Given the size of the minerals industry worldwide, and its complexity, it is surprising that, aside from the present authors, only one other NDM-style research project has been published in this area. However, it should be noted that other more general papers examining decision making in mining have been published (eg by Widzyk-Capehart et al. [3]). Until recently, the only comprehensive Human Factors text in this area was a US Bureau of Mines funded book by Sanders and Peay [4]. However, this was published before the NDM paradigm was commonly accepted, thus the approach does not rate a mention in that book.

The only previous research study found that specifically used the NDM paradigm in the minerals industry was

\footnotetext{
*Address correspondence to this author at the Minerals Industry Safety \& Health Centre, University of Queensland, QLD 4072, Australia; Tel: +61 7 3346 4087; E-mail: t.horberry@mishc.uq.edu.au
}

conducted by Dal Santo [5]. Her work investigated ground control (that is, rockfall prevention) decisions made by mining engineers working in underground mines. Her two main findings were the importance of situation assessment (that is, the ability of mining engineers to be able to 'read the ground') and how the characteristics of decision making changed not only with experience, but also with motivation, expectation and specific hazard knowledge. The key focus of Dal Santo's work was on improving the design of ground control training; it appeared that many of the tools of NDM (such as the CDM, discussed later) were of considerable benefit in understanding ground control decisions in mining, and developing better training based on this understanding.

However, some additional work is beginning to emerge in Australia using NDM-style approaches. This paper details two current applications of NDM in the minerals industry (all of which received human ethics approval by the University of Queensland, Australia). These two summary case studies will both present the information in a similar structure, first giving a short overall description of the area, then the purpose of the research, how NDM is relevant (for example, including what research methods are being employed), what is being done in the research and finally what are some of the indicative results.

\section{CASE STUDY 1: THE USE OF THE CRITICAL DECISION METHOD FOR MOBILE MINING EQUIPMENT INCIDENT ANALYSIS}

\subsection{Description}

Decision making is an important component of the work of mobile equipment operators in the minerals industry, where even the working environment is literally being physically altered every working day, decision making is real time and is distributed across radio communication. Therefore, it is unsurprising that a significant proportion of mining accidents, often costly and fatal, are in some way related to the operator misinterpreting cues from the environment or not having enough cues [6]. 
This section will describe the application of an NDMinspired technique - the Critical Decision Method (CDM) to investigate incidents related to mobile mining equipment. CDM occurs in 4 stages, known as 'sweeps', with a series of structured probes to construct the incident. Multiple sweeps of the incident or situation are made to progressively deeper understand the challenges operator's faced and strategies employed by decision makers to cope with these domain demands [2].

The first stage is 'incident identification and selection'. An appropriate incident is selected, if not already defined, and the participant is asked to give an account of the incident from start to end. In these situations it is recommended that tough cases involving non-routine tasks or situations and complex decisions be selected. They are more likely to assist in the elicitation of relevant tacit knowledge [7]. In the second stage, the information is grouped into relevant decisions or actions known as 'chunks' to create a timeline. The interviewee is encouraged to expand and correct information. In the third phase, the critical decisions are selected and examined in greater depth. As stated recently, the method:

\begin{abstract}
"...get inside the experts head and see the world through his or her eyes... What is the story behind the story? Based on the first two steps (the researchers) know what happened... but what did (the interviewee) know, when did they know it, how did they know, and what did they do with what they knew?" [2, pages 7879].
\end{abstract}

This is achieved by using a set of probe questions relating to, among other things, environmental cues, options, goals, experience and mental models. The fourth and final stage involves posing a number of hypothetical scenarios/changes to the event in the form of 'what-if' questions.

CDM is well suited to understanding incidents related to mobile mining equipment (such as haul trucks, shovels and bulldozers) where the decisions of experienced operators in a complex environment are often related to causing, or preventing, accidents. For example, Tichon successfully used CDM to elicit knowledge from train drivers, finding significant numbers of environmental cues, actions to be taken and possible errors [8]. However, CDM has used extremely rarely in mining and, aside from the authors' own work, no published specific CDM material are believed to exist relating to the role of mining equipment operators.

\subsection{Research Purpose}

Traditional incident identification techniques deal mainly with the identification of a sequence of events hoping to identify unsafe acts or conditions; what happened [9]. Some go beyond looking at causal analysis to identify the relationship between incident events and the breakdown of any controls; how it happened. However, it has been suggested that newer techniques are required to more greatly understand what factors influence and predispose the decisions of mobile equipment operators [5]. Better investigation techniques are needed to help understand the incident, and decisions, from the perspective of the person making those decisions to give an appropriate representation of why the incident occurred. The purpose of the current study was to see if CDM is an appropriate method to apply as an incident investigation method, and to assess what additional insight CDM brings compared to a leading incident investigation method used in the minerals industry.

\subsection{How NDM is Relevant}

It was hypothesized that NDM would better describe the cognition of operators mobile mining equipment under time and production pressure that often interact with other equipment in a minimally defined mine environment than more traditional investigation approaches. CDM (and NDM more broadly) assumes that persons were attempting to make sense - sensemaking - of the information in real world contexts [2]. It hopes not only to elicit and analyse but also represent this information. Furthermore, it is ideally suited to interpreting complex decision making in novel situations. It seems likely that complex and novel situations will be related to a significant number of the accidents that occur during operation of mining equipment.

Simply asking mobile mining equipment operators what they used to make decisions is unlikely to be enough. For example, people can learn to control and make decisions in complex situations without being able easily verbalise their thoughts and actions [5]. Therefore, in complex situations knowledge has to be specifically elicited from the persons involved. This is often called Knowledge Elicitation (KE). The CDM method attempts to provide scaffolding for KE. The underlying goal, and assumption, of CDM is that increased understanding of how people actually make decisions within various domains will allow future improvement of these decisions either through training or changes to environment, including equipment design. Therefore, it is likely that application of CDM will lead to greater understanding how mobile mining equipment operators make decisions to improve future equipment design, operator training and incident analyses methods.

\subsection{Scope of the Research}

The authors of this paper are currently involved in research to apply CDM with mobile equipment operators to examine past incidents. Given the in-depth process of a $\mathrm{CDM}$, the recommendations of other CDM researchers [2] and the remoteness and general difficulty making equipment operators available, large numbers of participants were not required. The goal was to investigate if CDM could provide valuable information about the decision making process of operators involved in incidents or near miss events. Therefore, the only criterion for involvement in this study was to be a mobile equipment operator who has been involved in an incident or near miss. Further details of the CDM in mining methodology are given in Horberry and Cooke [10].

Ten CDM interviews were successfully completed during site visits to surface coal mines in Australia. The following is a brief description of each incident and critical decisions that were made:

1. Engine fire in digger where the decision to press the fire suppression system was critical. 
2. Rollover of personnel carrier whilst on a decline of a mine haulage road where the decisions on speed, angle and part of the road driven on were critical.

3. Collision between bulldozer and grader when the grader was parked behind haul trucks waiting to be loaded by the digger. The decision on parking position of the grader and timing of radio contact were critical.

4. Loss of control of a haul truck on a down ramp not long after it had begun raining where the decision to continue driving in the rain and speed of descent were critical.

5. Uncontrolled drop of shovel bucket colliding with reversing haul truck where the judgement of 'normal' drop on the bucket was critical.

6. Rollover of bulldozer whilst cleaning overburden where the judgement of approach angle near a previous lower cut, and another bulldozer driver to make that cut, was critical.

7. Fire whilst filling a fuel truck at a fuelling station where the decision to evacuate and not press an automatic stop was critical, as fuel continued to be pumped into the fire.

8. Haul truck with dump tray up struck a reject chute bin where a critical assumption was made that maintenance personnel had fixed a problem with the tray and had lowered it.

9. Decision to perform maintenance when haul truck engine shows low horse power where decisions about likely causes were interestingly made with competing demands between operation and maintenance and trial and error process starting with the option that, if true, would limit downtime.

10. Missing alignment when reversing haul truck into shovel where the key cue affecting the decision was the alignment of the shovel in the side mirrors of the haul truck.

\subsection{Indicative Results}

\subsubsection{Specific Results}

To give more details of the findings from this process, one of the incidents is used as the example. This example is incident number 8: 'Drove Haul Truck with Dump Tray Up striking Reject Bin Chute'. A flowchart of the incident is shown in Fig. (1). This shows the major stages of the event (in text boxes) and the key decision points (as rectanglesafter boxes 3, 6, 11 and 13).

For the key decision points, the decisions actually made were further explored. In terms of the content of this examination, examples of what the deepening probes found in Sweep 3 and the "What if" inquiries in Sweep 4 included:

- "Idiot Balls" were placed around the mine, but the park up bay was past the last idiot ball wire.

- $\quad$ Driver had rarely started truck with tray up.

- Display for tray up was only visual.

- Display possibly obscured by sun glare.

- $\quad$ Chatting to auto-electrician during the drive.

- No visual feedback on tray from the driver's position.
- An audible signal might have alerted the driver to the fact the tray was up.

- If the driver was not friendly with the electrician he may have noticed the tray (due to him being partially distracted by their conversation).

- If the park up bay was further from the reject bin OR the road conditions were better, the driver would have reached $8 \mathrm{~km} / \mathrm{h}$ and set off the alarm.

\subsubsection{General Results}

The main findings of the research showed that CDM is a very useful tool to 'get in the head' and better understand the mindset of the personnel involved in incidents [10]. The method is of increasing value with more complex incidents. The research also found that CDM uncovered important details of the incident which were not included in current investigation reports - it is believed that such information could have a key benefit to help fully understand (and learn lessons from) the incident. As noted in the specific results section 2.5.1, one incident examined was a haul truck with its dump tray raised striking a reject bin chute. In this incident the CDM process found that a visual warning of the tray up was possibly obscured/made less conspicuous by sun glare; a potentially important factor that was not included in the previous incident report.

Overall, the CDM results highlighted four issues [10].

2.5.2.1. Mobile Mining Equipment Incidents are Regularly Complex

The CDM revealed many of the incidents related to mobile mining equipment to be complex in nature. These involved the alignment of a number of events, and the failure of numerous barriers of defense, commonly triggered by local atypical conditions. This aligns well with the James Reason model of the dynamics of organisational accident causation $[11,12]$ and supports the view that CDM should be a valuable tool to add to incident investigation. For example, in the above-reported incident where a bulldozer struck a grader, the immediate causes of unsafe acts involved the parking of the grader and loss of situational awareness by the bulldozer operator. However, upstream the local workplace factor of the design of traffic flow on site and the organisational factor of production pressure made the unsafe acts more likely to result in an incident.

\subsubsection{CDM Increases in Value with Complexity}

In general, the CDM interview process was able to establish a good understanding of the incident in most cases. In complex situations, the interview was successful in not only establishing the story of what happened but also the critical decisions made and the operator's sensemaking related to these decisions. With less complex events, involving simpler decisions, the first two stages of the CDM were helpful in establishing the circumstances surrounding the incident. However, if the decisions made were relatively straightforward using obvious environmental cues, the deepening and 'what if' probes did not add significantly to the understanding of the event.

For example, the latter stages of CDM for the fire in the digger example did not gain significant information because the cue of smoke and the action of pressing the fire 

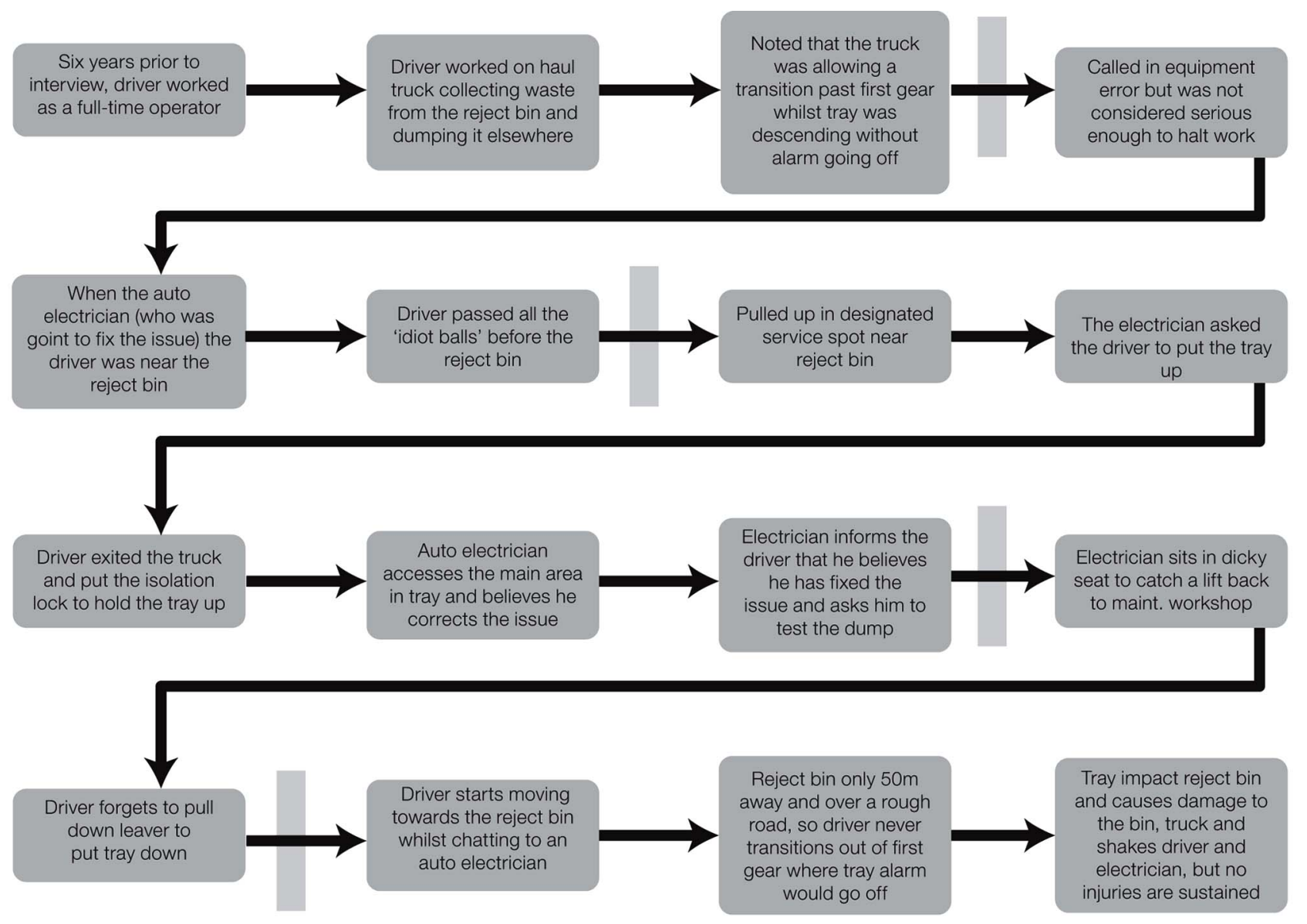

Fig. (1). Flowchart of incident number 8 (haul truck with dump tray up struck a reject chute bin).

suppression system was not a complex decision. In contrast, the incident where a haul truck lost control whilst descending a ramp appeared to be a simple case of excessive speed for the conditions at first. However, further probing using CDM found the decisions were significantly more complex, such as the team decision by different haul truck drivers on the same route on judging when wet weather makes conditions too dangerous.

\subsubsection{CDM Can Help Uncover Important Details Not in Site Investigation Reports}

For a number of the incidents the local site investigations in the form of standardised Incident Cause Analysis Method (I-CAM) reports were obtained. The I-CAM incident investigation method is based on the work of James Reason's models of organisational accident causation [12] and Jens Rasmussen's skill/rule/knowledge model of human error [13]. It could, therefore, be conceivably classified as a Human Factors technique. I-CAM provides a classification system for various local or latent factors that may be involved in an incident [14].

Upon reviewing the incidents, it was clear to researchers that the CDM interviews often identified information not contained in the I-CAM report. For example, in the incident where an overhead chute was impacted by a haul truck with the tray in the upright position, large colorful balls on wires - locally known as 'idiot balls' - were set below the height of the chute. These serve as height indicators for drivers, similar to chains on low bridges or entering suburban car parks. They would have usually been contacted before the chute if a tray was in the upright position. However, a designated park up position for the haul truck was located past these balls. In this park up location, the operator raised the tray for a maintenance task and either forgot to lower it or did not notice that it had failed to lower before driving towards the reject bin. The location of the park-up bay and the idiot balls were not included in the incident investigation. 
There are a number of other examples with similar oversights. Therefore, the provisional conclusion is that using the CDM method would assist in the data gathering for I-CAM and other incident investigations.

\subsubsection{CDM Can Help Understand the Organisational} Culture of a Site

During a number of interviews, it appeared that a site culture of viewing affected the interviewee's perspective of the incidents. Specifically, the interviewees generally appeared reluctant to consider the influence of a system and more likely to blame the actions of people, including their own. They were often heard to use phrases like 'I should have' or 'he should have' and drift into generalizations about what was required by a specified procedure. In one case the participant noted that he and another participant shared the blame for a collision by not establishing positive radio contact when, in fact, it is likely that the radio system was unusable in the situation. This reflects Dekker's 'Bad Apple Theory' of human error where failures are introduced into a system due to unreliable persons and corrected by tightening procedures [15]. Therefore, occasionally, it was difficult to get employees to investigate alternatives using phrases like 'that would never happen'. As such, CDM helped reveal that the prevailing site culture was partly a blame culture: human error was frequently cited as the 'cause' of incidents. A deeper understanding of the human system interactions was not attempted in previous investigations, nor was the organizational culture specifically examined.

\subsection{Future Work}

Future research is required to gain more evidence of the practical usefulness of CDM to assist in creating effective interventions. To achieve this, the researchers plan to conduct further work with CDM in two areas: employ it in an actual incident investigation (integrate, apply and evaluate $\mathrm{CDM}$ in an I-CAM investigation) and trial the technique's use as a proactive method, such as when introducing new controls or equipment (eg collision detection systems). An initial trial with the proactive CDM method has recently occurred [10] and the results are promising.

At the completion of this research it is anticipated that a substantive body of peer-reviewed evidence will have been developed. This will show that the detailed cognitive task analysis from an NDM perspective can assist in understanding the decisions made by mobile mining equipment operators. In turn, this will assist in making practical recommendation to improve safety, both reactively and proactively.

\section{CASE STUDY 2: NDM AND INTERFACE DESIGN IN MINERALS INDUSTRY PROCESS CONTROL}

\subsection{Description}

Process control systems are being increasingly used in mine sites to control grinding, smelting and flotation processes. However, many of these systems are not being used to their full potential. A recent industry survey indicated that, on average, over $60 \%$ of control loops are under performing [16]. Poor performance of these control loops inevitably results in unsatisfactory industry-wide production quality, such as high variability in product quality, reduced throughput and increased downtime. Interviews with domain experts in mineral processing revealed that one of the key reasons for system underperformance is that the control system information is not organized in such a way to support human controllers' work. This is reflected by a common phenomenon that most mineral process controllers tend to ignore the alarms most of the time in their control rooms. Thus, work to improve the interfaces (including alarms) of minerals industry process control systems is urgently needed.

\subsection{Research Purpose}

1. To enhance the performance of mineral processing production, an operator-centered approach to improve information design of the control systems is being undertaken to match the generalized process of Decision Centered Design (DCD) as described by Crandall et al. [2] and Huttton, Miller and Thordsen [17]. The DCD process was slightly modified to allow for a review and potential re-design of an existing system rather than the design of a new one. It involves five steps:

2. Preparation in order to gain an understanding of the domain, users and tasks. This includes identifying key complex tasks.

3. Knowledge Elicitation where Cognitive Task Analysis methods are used to understand how key decisions are made.

4. Analysis and Representation where data is decomposed into discrete elements in order to identify user decision requirements and central themes and issues. This reveals key 'leverage points'.

5. Application and Design where knowledge about determining how to best support decision making is translated into design concepts and prototypes.

6. Evaluation where the new designs are tested and the impact estimated.

Very little work specifically using the DCD process has previously been done in mining. The first step of this research involved visits to several mineral processing plants to understand the process controllers' work. Of particular interest was how operators use the information both "in" and "around" the control room to make decisions. It is anticipated that a 'gap' between the operator's cognition (information) needs and the support from the operator interface would be identified. Therefore, it would be possible to gain some or all of the lost production potential, noted in Section 3.1, by redesign of existing interfaces and better design of interfaces on new systems.

\subsection{How NDM is Relevant}

NDM research studies human cognition in real world, complex, dynamic and uncertain conditions. Particularly, NDM emphasizes that rapid categorisation of a situation enables people to make efficient decisions by comparing them to, at most, a small number of possible options and taking action when an adequate match is found. The dynamic work environment of mineral process control certainly fits into this category. This categorisation is significantly impacted by the work context, in this case, the mineral 
processing operating environment, the task prescribed to mineral process controllers and their levels of training and experience. Understanding how these different elements affect operator decision making is vital to improve outcomes through altering how information is presented to operators. Obtaining in-depth insights of the human cognition (information) needs for managing their work is one of the key challenges raised in this work. Therefore, CDM methods based on NDM models are the ideal data collection and knowledge elicitation tools to help study experienced mineral process controllers at different mineral processing plants.

\subsection{Scope of the Research}

To date, two plant visits at different types of mineral processing facilities have taken place: to copper processing and gold processing plants. For safety and productivity reasons, it was always essential in this domain that the study should not to interfere with operators' work. In addition, the numbers and responsibilities of operators working in the control room varied in these plants. Therefore, this research needed to work around these constraints. Thus, the operators' views or comments were mainly collected during the breaks in operational tasks.

However, the methods used still focused on how the key decisions were made within a DCD framework. NDMrelated data collection or knowledge elicitation methods, required for the second stage of DCD, were adapted to fit around the flexible and uncertain work environments in different types of mineral processing plants. The primary methods used to date in plant studies include naturalistic/field observations and a short in-situ interviews, based on the CDM. Often the interview broke into several sessions depending on the availability of the operator.

In general, the purpose of the observation was to discover how the operator detected and interpreted the information available in the control room to identify if the situation required human intervention and how their control decisions were made. Thus, the observation focused on how the operator handled the plant incidents or disturbances. After the incident was resolved and when the operator was free, a follow-on short CDM interview was conducted. The interviews probed how operators made key decisions in a class of incidents, including identification of important cues, plausible goals, decision points and typical operational strategies.

Twenty operators were observed for approximately 100 hours and the majority of them were also interviewed. The participants ranged from new operators, who worked in the plant for up to eight month, to experienced operators with 30 years work experience in this industry. Further details of the methodology used are given in Li et al. [18].

\subsection{Indicative Results}

The first stage in DCD involves gaining an understanding of the domain. This was achieved by means of two plant visits. For the scope of this paper, only a brief illustration of the domain (that is, operator's role in minerals processing) is provided.

\subsubsection{Plant Function}

Essentially, minerals processing is about mechanically breaking up mined ore until it is of small enough size that the valuable mineral can be chemically separated from the unwanted/waste material. The processing of minerals is complex, involving many steps, multiple pieces of equipment and sorting of minerals. This includes the processes of crushing, grinding and flotation. Equipment used in these processes includes crushers, Semi-Autogenous Grinding (SAG) mills, ball mills, cyclones and flotation cells. The production process is not a simple linear progression. Material is divided throughout the process and sent forward or backward to different areas of the plant based on various separation criteria.

The information about plant function was obtained through plant visits and by studying flow sheet diagrams. Additionally, the basic control philosophy of the grinding and flotation process was elicited from the short CDM-type interview with operators and metallurgists.

\subsubsection{Human Control Tasks}

Though some of the process is semi-automated, operators are continually monitoring and ideally making adjustments in an attempt to stabilize and optimize plant performance. Therefore, the control information required by operators is needed to ensure that their tasks are successfully integrated into the overall production system process. Human control tasks are usually determined by system complexity and staffing levels in the control room. As the two mineral processing plants under investigation were equipped with different control system technologies and different job allocations in the control room, the operational tasks were not the same. For example, in one plant, the flotation circuit was largely manually controlled; the flotation controller ran regular field sample check in flotation cells every four hours to monitor the production status. At the other plant, the flotation controller used the information from the online mixed stream analysis tool in the control room for monitoring the production quality in the flotation cells and he only occasionally ran the field manual sample check.

\subsubsection{Supporting Information}

Information is directed towards operators from various sources with the aim of supporting them to make appropriate judgments in their prescribed tasks. The information support in the control room includes the screen layout and the information content and styles from the displays and alarms, the setting of the TV (camera) monitors, and the information on a whiteboard. The operators mainly used various overview displays from different control systems and CCTV on their control desk to maintain their situational awareness of the plant status. In this case, their attention was primarily triggered by alarms. Most of this information was collected through observations and the CDM-type interview.

The analysis of the plant function, human control tasks and supporting information together allows analysis of how the mineral process controllers perceive, analyse, interpret and act on the information from the control systems to support their work. The information gathered also allowed 
an understanding of the domain to be gained. It was then possible to move to the knowledge elicitation step of DCD with the aim of identifying key decisions. It has been suggested that this stage, and DCD in general, should focus on 'tough cases' because these reveal the greatest knowledge about where a system is underperforming in the time available [2]. However, as is common in field observations using Cognitive Task Analysis methods, though the observation of operators can yield significant information to understand the work domain, only a few actual incidents and critical decisions occurred in this study. Therefore, it was essential to extend a single event to a representative type of plant incidents in the interview.

For example, in one incident, significantly larger than ideal rocks came into the SAG mill which drove the power over the upper limit. Trend graphics of the plant performance during the period of the critical incident were used as the tool to help interviewees recall the event. This incident was extended to monitoring and reacting to variability of the size, shape and density of rocks fed into the mill. Thus, more data about the operational strategies and the critical cognitive decision points for dealing with various types of input to the SAG mill could be identified through interviews. In addition, an interview with the plant control engineer (an expert metallurgist) provided an alternative perspective towards understanding of operator's decision making performance. Interestingly, the metallurgists had their own views on what control strategies should be and their likely impact on plant performance.

The findings to date provide fruitful insights to many topics including the assessment of the current operator interface (displays and alarms), the investigation of operator training and better understanding operator's work needs. Table 1 provides an illustration of the selected data obtained during the investigation.

\subsection{Future Work}

Though the work is ongoing, data collected to date show that a DCD approach, using methods inspired by NDM, has been successfully adapted to understand the novel, flexible and uncertain work undertaken by process control operators in mineral processing domain. The next step of the research will continue the knowledge elicitation step of DCD, using CDM methods, potentially incorporating elements of work domain analysis [19]. This will include involving more operators in full CDM interviews to probe the issues, such as the difference between the experienced and the new operator.
The work will also continue to focus on tough cases as DCD has already proven to be a valid method for understanding minerals processing control.

Subsequently, it is hypothesized that key information requirements at leverage points can be represented, leading to system modifications and eventual productivity improvements [18]. In summary, work to date shows that methods to understand the decision making of minerals processing operators using NDM models can be effective. Future work aims to translate this knowledge into redesign of process control interfaces and thus ultimately to help improve system performance.

\section{CONCLUSIONS: THE FUTURE OF DECISION MAKING RESEARCH IN THE MINERALS INDUSTRY}

\subsection{Discussion}

The two case studies presented here examined different aspects of the minerals industry. The key researchers involved in each were from different backgrounds (ergonomics, risk management and cognitive engineering) and the outputs being produced were very diverse (analysing mobile equipment incidents or developing better process control interfaces). However, the glue that binds them both together is an operator-centered perspective, in particular, using NDM approaches and methods.

In both case studies, the NDM paradigm is evident in the need to elicit knowledge from experts in the field (for example, using the CDM technique). NDM is also important to help to design equipment interfaces or incident analysis techniques that have an explicit focus on the actual decisions made by operators in demanding situations arising from the complex sociotechnical minerals industry system in which they are working.

\subsection{Key Findings}

Although ongoing research, key findings have emerged in the two areas. The use of the CDM technique in the analysis of mobile equipment incidents has shown that the knowledge elicited from the operators involved in these events provided valuable 'extra' information compared to the incident analysis methods currently in use. By focusing on the key decision points for operators and unpicking the cues, information, goals, prior experience and related probes, the research was able to obtain a more detail description of the incident event than the standard narratives used in much of the minerals industry today [10]. For example, the current

Table 1. Sample Data from the Two Mineral Processing Plants

\begin{tabular}{|c|c|c|}
\hline Topics & Associated Problems & Observed Examples \\
\hline \hline Operator display & $\begin{array}{c}\text { Poor support of the operator's } \\
\text { situational awareness of critical } \\
\text { process dynamics }\end{array}$ & $\begin{array}{c}\text { Operators rely on guesswork in what was happening in the SAG mill, such as why the power } \\
\text { and baring pressure fluctuates. It was hard for them to predicate how the fluctuation would } \\
\text { impact on production quality. }\end{array}$ \\
\hline Alarms & $\begin{array}{c}\text { Alarm: Inadequate prioritization to } \\
\text { direct operator attention }\end{array}$ & $\begin{array}{c}\text { The operator read through two pages of the alarm list and only picked up one critical alarm } \\
\text { which would trip off the slug mill, if not responded to. However, this critical alarm was } \\
\text { "hidden" among the 50 nuisance alarms. No distinction is given to critical alarms. }\end{array}$ \\
\hline Operator training & $\begin{array}{c}\text { Lack of training of using trends } \\
\text { graph }\end{array}$ & $\begin{array}{c}\text { Only 6 operators were observed to use trends to analyse the performance of the SAG mill } \\
\text { over time. The rest of the operators claimed that they seldom use or don't know how to use } \\
\text { these trends in their work. }\end{array}$ \\
\hline
\end{tabular}


technique used for the investigation of an incident involving a road grader and a bulldozer found that breaching procedures and not establishing radio contact between operators was the primary 'cause' of the accident. Therefore, a reminder to operators to follow procedures about radio contact was the sole action taken to prevent a repeat collision. However, the CDM methodology identified that the background noise of the bulldozer and the hearing protection worn by the operator might have prevented the operator from hearing and responding to radio calls from other operators in the vicinity, and thus positive radio contact could not be established in this situation.

Similar findings are being obtained in the process control case study regarding the importance of information currently displayed to the operators through the interface. This is especially an issue when the plant was in an abnormal state and the operator was trying to diagnose the problem. The plethora of alarms being activated, which were largely ignored by the operators, is a good example of why the interface requires redesign from an operator-centered perspective, particularly focusing on the emergency, 'difficult case' or other critical events [18].

Admittedly some of the findings emerging from the two case studies might also have been obtained by other Human Factors, Risk Management or Cognitive Engineering methods (for example, using Cognitive Work Analysis (CWA) [19]. But the strengths of the NDM approach and associated tools, such as CDM, are that they can be easily combined with other techniques (eg other observational methods, incident data or Human Factors audits) and that they can be significantly quicker to complete than rival methods such as Cognitive Work Analysis. In the minerals industry, the time available to collect data is often extremely limited and needs to be flexible to fit around existing work processes. Extensive study of the work domain for several months is usually impossible, hence the NDM approach that was developed to study human performance in the field seems a logical, valid and robust approach to this domain.

\subsection{Recommendations for Future Decision Making Research in the Minerals Industry}

Hopefully this paper has demonstrated some of the richness of the issues related to human performance and decision making in the minerals industry. In a discipline largely unexplored from a human-centered perspective, it has shown how NDM-inspired approaches and tools can be successfully deployed in several disparate case study areas.

Given the lack of previous NDM work in this domain, there is plenty of scope for adapting many of the developed methods (e.g. CDM) to the minerals industry. Clearly, by focusing on decision making in real environments, NDMstyle approaches should be applicable to many issues that involve people working in the minerals industry. In more depth, and inspired by Horberry, Burgess-Limerick and Steiner [20], future related work in this domain might include the following:

- Refining data collection methods and tools. As mentioned earlier, the amount of time to collect human-related data in mining can be extremely limited. Flexible, reliable, valid and quick methods are needed, (but obtaining a full understanding of a task or environment still often requires observing an operator). An example is a shortened version of CDM and/or being able to split the CDM session into several 'chunks' to fit with an operator's free time. A second example concerns studying operator decision making in the field to develop risk management tools before starting and whilst undertaking high-risk tasks [21].

- $\quad$ Looking deeper at human error in mining incidents to understand how and why they occurred. The label 'Human Error' explains nothing in itself - it does not show what caused the error, what could have been done to prevent it or what measures could be put in place to limit the occurrence of similar errors in future [6]. The move away from the 'train and blame' view will help to generate a better understanding of the human element in incidents and will ultimately help develop safer human-machine systems. The knowledge elicitation approaches and emphasis on design for critical events are firmly within this framework.

- Considering issues around motivational and emotional aspects of a task, work process or interacting with a piece of equipment, as these can have a strong influence on performance (e.g. equipment misuse or work motivation). As with NDM research in other domains [22], the emphasis on such aspects in the minerals industry is important.

- $\quad$ Finally, helping develop a greater awareness by all stakeholders in this industry (e.g. managers, designers, contractors, regulators, operators and maintainers) of the benefits of applying a usercentered approach in mining. This should lead to an increased professional recognition in the minerals industry of the status of a Human Factors expert, Cognitive Engineer or User-Centered Designer.

In this way, focusing on how decisions are actually made by experts and other operators in the field, then designing effective mining equipment, tasks, procedures and training to take these into account will be key challenges over the next 10 years.

\section{ACKNOWLEDGEMENTS}

The authors wish to acknowledge the organisations and operators involved in this work. In addition, we thank colleagues at the University of Queensland (Australia) and the reviewers of this paper.

\section{CONFLICT OF INTEREST}

None declared.

\section{REFERENCES}

[1] Klein G. Naturalistic decision making. Hum Fact 2004; 50 (3): 456-60.

[2] Crandall B, Klein G, Hoffman R. Working minds - a practitioners guide to cognitive task analysis. Cambridge, MA, USA: Massachusetts Institute of Technology 2006.

[3] Widzyk-Capehart E, Hill R, Sivadorai M, Sheppard G, Jessett A Rope shovel hoist stall prediction and operator tactile feedback. In: Proceedingds of $5^{\text {th }}$ International Symposium on Mine Planning \& Equipment Selection MPES 2006, 21 - 23 September2006; Turin, Italy. 
[4] Sanders MS, Peay JM. Human factors in mining (IC 9182). Pittsburgh, PA: U.S. Department of the Interior, Bureau of Mines 1988.

[5] Dal Santo L. Decision-making in the mining industry: an examination of risk-related judgments and decisions in the area of ground control, and the implications for training mining engineers. Unpublished PhD thesis, University of Queensland, Australia 2005.

[6] Simpson G, Horberry T, Joy J. Understanding human error in mine safety. Aldershot, UK: Ashgate Press 2009.

[7] Shadrick S, Lussier JW, Hinkle R. Concept development for future domains: A new method of knowledge elicitation. Fort Knox, Kentucky, USA: US Army Armor Center 2005.

[8] Tichon J. The use of expert knowledge in the development of simulations for train driver training. Cognit Technol Work 2007; 9(4): 177-87.

[9] Doytchev DE, Szwillus G. Combining task analysis and fault tree analysis for accident and incident analysis: A case study from Bulgaria. Accid Anal Prev 2009; 41(6): 1172-9.

[10] Horberry $T$, Cooke $T$. Using the critical decision method for incident analysis in mining. J Health Saf Res Pract 2010; 2(2):1023.

[11] Reason J. Human error. Cambridge, UK: Cambridge University Press 1990.

[12] Reason J. Managing the risks of organizational accidents. Aldershot, UK: Ashgate 1997

[13] Rasmussen J. Skills, rules, knowledge; signals, signs, and symbols, and other distinctions in human performance models. IEEE Transactions on Systems, Man and Cybernetics 1983; 13: 257-66.
[14] De Landre J, Gibb G, Walters N. Using Incident Investigation Tools Proactively for Incident Prevention. Australian \& New Zealand Societies of Air Safety Investigators Conference 2006; Melbourne, Australia.

[15] Dekker S. The field guide to understanding human error. Aldershot, UK: Ashgate 2006

[16] Thwaites P. Process control in metallurgical plants: towards operational performance excellence. Automining 2008 International congress in automation in mine industry, Santiago, Chile.

[17] Huttton RJB, Miller TE, Marvin L. Decision-centered design: Leveraging cognitive task analysis in design. Hand book of cognitive task design. In: Hollnagel E, Ed. Mahwah, NJ: Lawrence Erlbaum \& Associates 2003.

[18] Li X, McKee DJ, Horberry T, Powell M. The control room operator: the forgotten element in minerals process control. Minera Eng 2011; doi:10.1016/j.mineng.2011.04.001

[19] Vicente KJ. Cognitive work analysis: toward safe, productive, and healthy computer-based work. Mahwah, NJ: Lawrence Erlbaum \& Associates 1999.

[20] Horberry T, Burgess-Limerick R, Steiner L. Human factors for the design, operation and maintenance of mining equipment. USA: CRC Press 2010.

[21] Marling G, Horberry T. Operator decision making before starting and while doing an activity/task. In: Proceedings of $9^{\text {th }}$ Naturalistic Decision Making Conference (NDM9), London, June 2009.

[22] Mosier KL, Fischer UM. Does affect matter in naturalistic decision making? In: Proceedings of $9^{\text {th }}$ Naturalistic Decision Making Conference (NDM9), London, June 2009

(C) Horberry et al.; Licensee Bentham Open.

This is an open access article licensed under the terms of the Creative Commons Attribution Non-Commercial License (http: //creativecommons.org/licenses/ by-nc/3.0/) which permits unrestricted, non-commercial use, distribution and reproduction in any medium, provided the work is properly cited. 